\title{
Shedding (Gamma) Light on the Cosmic Ray Population in the Galactic Center Region
}

\author{
Sofia Ventura* \\ University of Siena \& INFN, Pisa \\ E-mail: sofia.venturaepi.infn.it \\ Dario Grasso \\ INFN, Pisa \\ E-mail: dario.grasso@pi.infn.it \\ Antonio Marinelli \\ INFN, Pisa \\ E-mail: antonio.marinelli@pi.infn.it
}

The nature of the very-high-energy gamma-ray diffuse emission measured by H.E.S.S. in the Galactic Center (GC) region is still matter of debate. Two main scenarios have been proposed to interpret H.E.S.S. results, where the emission arises from a cosmic-ray (CR) population, which is either originated from a local PeVatron or is the "tip of the iceberg" of an inhomogeneous Galactic $\mathrm{CR}$ sea. The latter scenario is motivated by recent analysis of the Galactic diffuse emission measured by Fermi-LAT as well as from Milagro and recent HAWC results. In this contribution we compare these interpretations against H.E.S.S. data in combination with updated Fermi-LAT data, which allow to extend the measured spectrum down to few GeVs. Besides the Central Molecular Zone and the Sgr B gas complex - for which we find a good agreement with data for both scenarios - here we consider, for the first time in this framework, the emission of J1741302, under the hypothesis that is due to the interaction of CRs with a massive cloud lying on the Galactic plane at about $260 \mathrm{pc}$ from the GC. We find the CR sea scenario reproduces the spectrum of J1741-302 (+ Fermi-LAT ) remarkably well, while the GC PeVatron - due to the $1 / r$ profile of the CR density profile - falls short. Therefore, our result strongly favours the CR sea interpretation of the measured $\gamma$-ray diffuse emission from the innermost region of the Galaxy.

36th International Cosmic Ray Conference -ICRC2019-

July 24th - August 1st, 2019

Madison, WI, U.S.A.

${ }^{*}$ Speaker. 


\section{Introduction}

The Galactic Center (GC) is one of the most interesting regions for high energy astrophysics, and represents the perfect laboratory to study phenomena and physical processes that may be occurring in many other galactic nuclei. Among the reasons making that region a promising $\gamma$-ray emitter, arises the presence of a supermassive black-hole - coincident in position with the compact radio source Sgr $\mathrm{A}^{*}$ - that might have undergone violent activity in the recent past. Moreover, the evidence of starburst regions that may power intense cosmic ray (CR) injection, and a plausible cusp in the cold dark matter density that may strongly amplify WIMP annihilation.

Here we are more interested in the diffuse $\gamma$-ray emission that may shed light on the CR population in that peculiar region of our Own Galaxy. Noticeably, the gas complex in the GC is the only Galactic system, where the $\gamma$-ray diffuse emission has been observed above the TeV.

Indeed, following a first detection reported in [1] the High Energy Stereoscopic System (H.E.S.S.) collaboration recently confirmed the discovery of an intense diffuse emission from the Galactic Ridge (GR) region - roughly corresponding to the central molecular zone (CMZ) - a massive structure rich in molecular gas, which extends up to $\sim 250 \mathrm{pc}$ away from the GC along the Galactic plane (GP) [2, 3]. The nature of such emission is expected to be hadronic due to its tight correlation with the gas distribution and the strong losses suffered by electrons in the GC environment. The spectrum of the emission, which was measured from $200 \mathrm{GeV}$ to $45 \mathrm{TeV}$, is compatible with a single power law with index $\Gamma_{\text {ridge }}=-2.28 \pm 0.03_{\text {stat }} \pm 0.2_{\text {sys }}$, and a differential flux at $1 \mathrm{TeV}$ of $\Phi=1.2 \pm 0.04_{\text {stat }} \pm 0.2_{\text {sys }} \times 10^{-8} \mathrm{TeV}^{-1} \mathrm{~cm}^{-2} \mathrm{~s}^{-1} \mathrm{sr}^{-1}$. This is significantly more intense and harder than expected assuming the spectrum of the $\mathrm{CR}$ population in the $\mathrm{GC}$ region to be the same observed near the Earth, which according to latest AMS results has a spectral index close to -2.7 (for $\mathrm{CR}$ protons) above $300 \mathrm{GeV} / \mathrm{n}$ [4]. This fact, together with the reconstructed CR density profile peaking at the GC, has led the H.E.S.S. collaboration to argue that the primary particles responsible for such emission are originated by an accelerator - a PeVatron, since the primary proton spectrum must extends up to $0.4 \mathrm{PeV}$ at least at $95 \%$ C.L. - at the position of SgrA*.

A different interpretation of the same experimental results, however, has been proposed in [6], where the measured spectrum was also extended down to few GeVs using PASS8 Fermi-LAT data. In that scenario the CR large scale population, which hereafter we call the $C R$ sea, varies with the Galactocentric radius $R$, getting harder as $R$ decreases. Such dependence is motivated by several independent analyses of Fermi-LAT data showing the spectral index of the $\gamma$-ray diffuse emission to increase from $\Gamma \sim-2.7$ to $\sim-2.3$ for $R$ decreasing from 10 to $0 \mathrm{kpc}$ (though with large uncertainties/fluctuations in the innermost $2 \mathrm{kpc})[8,9,10]$. It was shown that such a behaviour can be described by a phenomenological model (gamma model) assuming a radial dependent diffusion coefficient, which was implemented with the DRAGON code [7]. The model also accounts for the CR proton and Helium spectra hardening at $300 \mathrm{GeV} / \mathrm{n}$ found by AMS-02 [4] introducing a corresponding feature in the injection spectra of those species.

Amazingly, the same model reproduces the diffuse emission observed by H.E.S.S. and FermiLAT in the GR region meaning that the CR population in that region is mainly determined by the large scale Galactic CR sea and that a PeVatron at the GC may not be required. Although this model does not predict the peaked CR energy density profile $w_{\mathrm{CR}}(R)$ for $R \rightarrow 0$, which was claimed 
by the H.E.S.S. collaboration ${ }^{1}$, it should be noticed that $w_{\mathrm{CR}}$ can only be measured indirectly and that H.E.S.S. data could be reproduced accounting for a larger-than-expected gas density in the innermost few parsecs of the Galaxy.

Taking in mind the large uncertainties in the gas distribution at the GC, a more promising strategy to discriminate the right scenario is to study the emission of clouds at the rim or outside the CMZ. Indeed, while the PeVatron scenario predicts $w_{\mathrm{CR}} \propto 1 / R$, hence a dim emission of relatively distant clouds, in the hard CR sea scenario $w_{\mathrm{CR}}(R) \simeq$ const., hence predicting a hard and intense emission from those farther objects too.

\section{The diffuse emission spectrum from the $\mathrm{CMZ}$}

The central molecular zone (CMZ) is one of the densest region and an asymmetric layer of the galactic interstellar environment [15] offering a thick target for CR proton and Helium collisions. Its mass is about $10^{5} M_{\odot}$ with an average density of $10^{2} \mathrm{~cm}^{-3}$ and extends up to $\sim 250 \mathrm{pc}$ away from the GC along the GP.

H.E.S.S. has measured its diffuse emission above $200 \mathrm{GeV}$ in a region delimited by the Galactic coordinates $|l|<1.0^{\circ},|b|<0.4^{\circ}$ excluding a inner circular region of angular radius of $0.45^{\circ}$. Here we use about 10 year of Fermi-LAT data ${ }^{2}$ to extend down to few $\mathrm{GeVs}$ the measurement of the diffuse emission spectrum in the same region. Several point-like sources are present in the region that have to be subtracted from the total emission. Here we use the Fermi Lat 8 Years (FL8Y) list of sources [5], which has 8 sources in that region and was built assuming the diffuse model gll_iem_v06 described in [9]. The FL8Y source list is based on the first eight years of science data of Fermi-LAT reconstructed with the PASS8 algorithm. We do not use the more recent 4FGL catalogue that is based on a not yet fully described background model. The H.E.S.S. and FermiLAT data points are reported in Fig. 1. We notice the good agreement between the two datasets, which overlap at few hundred GeVs and can actually be fitted with a single power law with index $\Gamma_{\mathrm{GR}}=-2.36 \pm 0.03$.

In the same plot we also show the predictions of the gamma model with and without (dashed line) the contribution of the CMZ. The latter represents our astrophysical background model since other diffuse backgrounds - as that due the isotropic extragalactic emission and the Fermi bubble are subdominant in that region. We checked that such background is basically coincident with the high-energy extrapolation of the gll_iem_v0 6 Fermi-LAT diffuse model used to built the point sources list as need for the consistency of our approach.

In the CMZ the model adopts a three-dimensional gas distribution based on CO and CS radio emission lines and velocity dispersion observations, and a detailed dynamical model [11]. We found a good agreement between that model and the hydrogen mass distribution used by the H.E.S.S. collaboration adopting a value of the $\mathrm{CO}$ to $\mathrm{H}_{2}$ conversion factor $X_{\mathrm{CO}}^{\mathrm{GC}} \simeq 0.65 \times$

\footnotetext{
${ }^{1}$ Notice however that a uniform density was assumed along the line of sight crossing of the CMZ. That approximation may result in an overestimation of CR density in the innermost few parsecs of the GP.

${ }^{2}$ To extract Fermi-LAT data we use the Fermi Science Tools v11r5p3 [14] with the event class CLEAN. We apply the recommended quality cuts (DATA_QUAL==1) \&\& (LAT_CONFIG==1), and the Fermi-LAT response function P8R2_CLEAN_V6.
} 
$10^{20} \mathrm{~cm}^{-2} \mathrm{~K}^{-1} \mathrm{~km}^{-1} \mathrm{~s}$ that is in good agreement with a similar value inferred from astronomical observations.

We repeat the same exercise for a sub-region at the rim of the CMZ, approximatively centered on the Sgr B complex that was also observed by H.E.S.S. [12].

From Fig. 1 the reader can see that for both the CMZ and the Sgr B regions the gamma model provides a good description of the data.

We stress here the relevance of Fermi-LAT data in both regions: indeed at few GeVs the gamma model predicts the same emission of conventional models assuming a uniform CR spectrum in the whole Galaxy hence providing a safe evidence that at those energies the emission is dominated by the CR sea. Therefore, the absence of a pronounced feature at higher energies is an evidence against the presence of a new component in the CR population in that region.

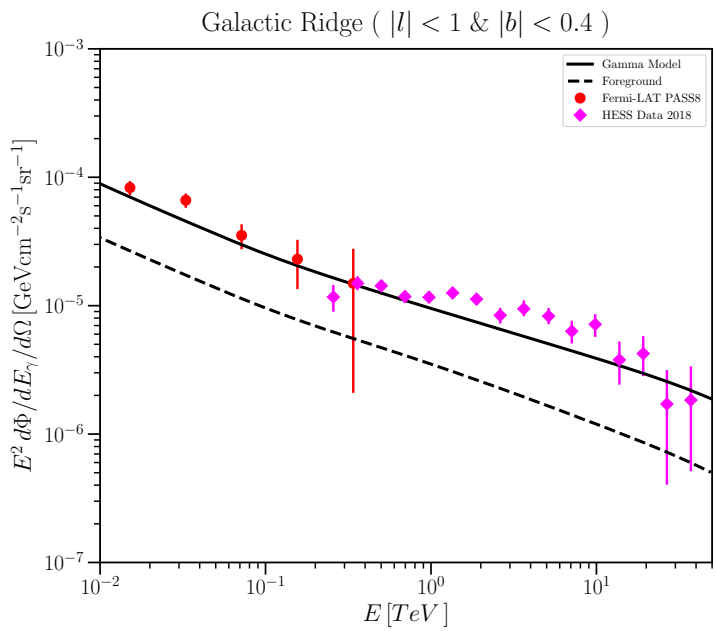

(a)

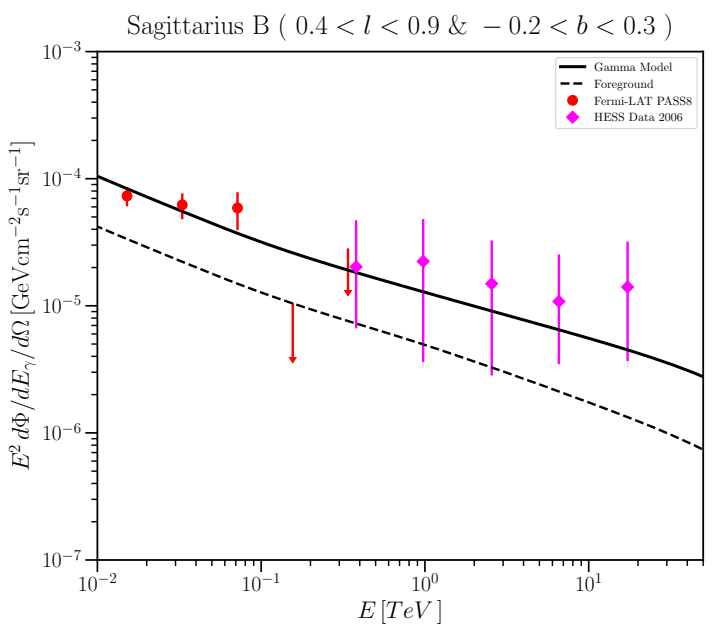

(b)

Figure 1: The diffuse emission spectrum of two different regions in the CMZ are reported. H.E.S.S. data for the Galactic ridge region (left panel) are taken from [3] while for Sgr B (right panel) from [12]. FermiLAT spectral points for both regions represent the excess counts derived in that analysis using 10 years of PASS 8 data and subtracting the contribution of point-like sources in the FL8Y source list [5]. The solid lines represent the total diffuse emission spectrum computed with the gamma model accounting for the contribution of the diffuse background computed on the basis of the same model (dashed line) .

\section{Going beyond the CMZ: the J1741-302 emission}

J1741-302 is an energetic $\gamma$-ray source recently discovered by H.E.S.S. [13]. It lays on the GP at angular distance $l=-1.7^{\circ}$ from the GC. The observed spectrum extends from 0.1 to $10 \mathrm{TeV}$ following a single-power law with index $\Gamma_{\mathrm{J} 1741-302}=-2.3 \pm 0.2_{\text {stat }} \pm 0.2_{\text {sys }}$ with no evidence of a cut-off. No clear astrophysical counterpart was identify for that source. Among several plausible origins of its emission, the H.E.S.S. collaboration proposed a massive molecular cloud (cloud 8), at a distance of $260 \pm 40 \mathrm{pc}$ from the GC, as one of the most likely. The mass of that cloud - derived from the $\mathrm{CO}$ emission line - was estimated to be $6.8 \times 10^{4} M_{\odot}$. 


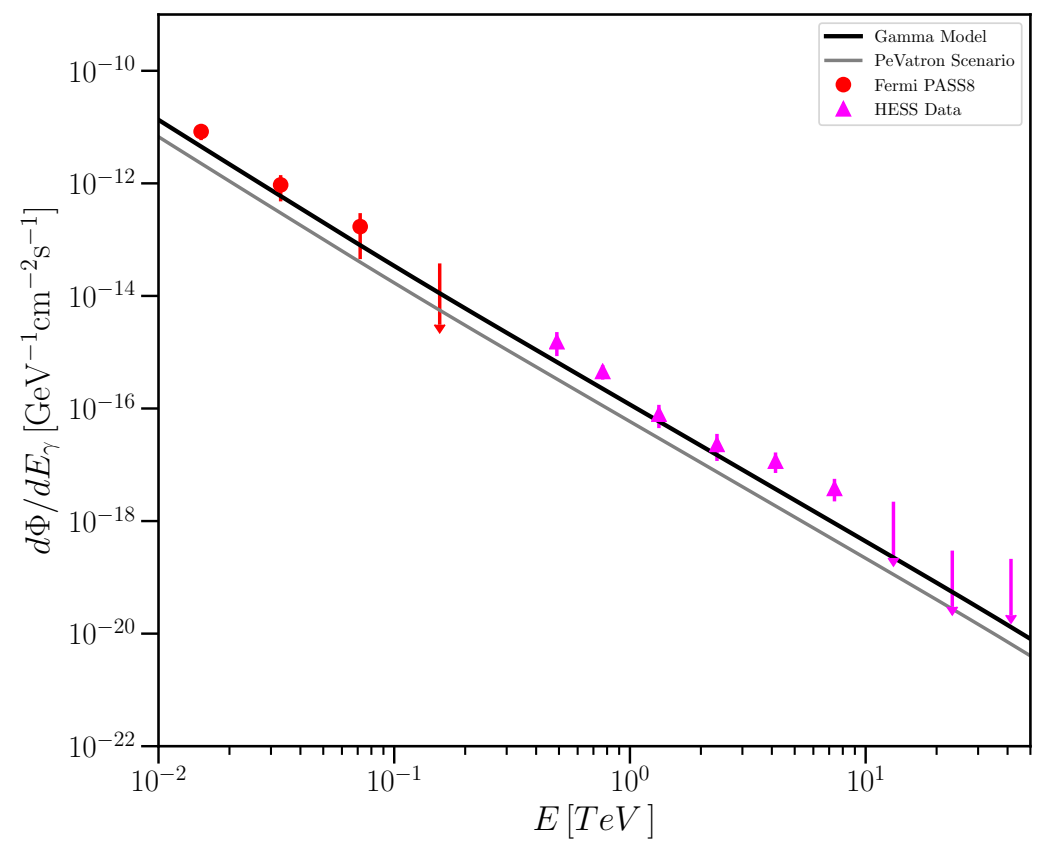

Figure 2: The $\gamma$-ray spectrum at the Earth from a circular region of angular radius $0.1^{\circ}$ centered on the position of J1741-302. H.E.S.S. data are extracted from [13], while Fermi-LAT spectral points are derived in the present analysis and represent the excess counts. The solid line is the prediction of the gamma model under the hypothesis that the emission is originated by the interaction of the Galactic CR sea with the cloud 8 (at $R=260 \mathrm{pc}$ ) identified in [13]. The gray line represents the prediction of the PeVatron model derived extrapolating the CR density profile reported in [2] to the position of cloud 8.

The very existence of such cloud is what most interest us. Indeed, that object is a natural target to probe how / if the CR population properties change with $R$.

To increase our lever arm, similarly to what done for the CMZ complex and Sgr B, we use Fermi-LAT data to extend the measured emission spectrum to low energies. We consider a region of interest of radius $0.1^{\circ}$ centered on J1741-302. Interestingly we found that also for this source the spectrum measured by H.E.S.S. extends steadily down to few GeVs. The spectral index we find combining H.E.S.S. and the derived Fermi-LAT data points is $\Gamma_{\mathrm{J} 1741-302}=-2.24 \pm 0.08$ that is in agreement with the gamma model prediction. Encouraged by this finding we then performed a more detailed comparison of those data with the prediction of the PeVatron and gamma model. As shown in Fig.2 we found that the gamma model prediction matches the observed spectrum remarkably well.

A rough check can be performed with a simple analytical computation as well. Starting from the Eq. (1) in [2] we find

$$
F_{\gamma}\left(\geq E_{\gamma}\right) \approx 4.6 \times 10^{-10}\left(\frac{M_{5}}{d_{\mathrm{kpc}}^{2}}\right) w_{\mathrm{CR}}\left(\geq 10 E_{\gamma}\right) \mathrm{erg} \mathrm{cm}^{-2} \mathrm{~s}^{-1}
$$

where $M_{5}$ is the cloud mass in units of $10^{5} M_{\odot}, d_{\mathrm{kpc}}$ the distance in unit of kpc. Adopting that for the cloud $8, M_{5} / d_{\mathrm{kpc}}^{2}=0.0094$, and for the gamma model, $w_{\mathrm{CR}}(\geq 4 \mathrm{TeV}) \simeq 0.1 \mathrm{eV} / \mathrm{cm}^{-3}$, we get 


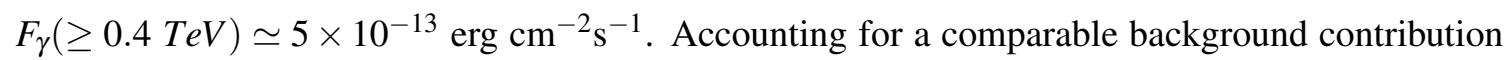
(see Fig.2), the inferred flux is very close to that measured by the H.E.S.S. collaboration, which is equal to $1.2 \times 10^{-12} \mathrm{erg} \mathrm{cm}^{-2} \mathrm{~s}^{-1}$.

For comparison we estimated the emission that should be originated by the same cloud if that would be illuminated by the $\mathrm{CR}$ population produced by a PeVtron at the GC position. Adopting the CR density profile reported by the H.E.S.S. collaboration in [2] we inferred that according to their results: $w_{\mathrm{CR}}$ is less than half the value predicted by the gamma model at the position of cloud 8 implying an emission significantly lower than one observed. This finding validates our hypothesis: the emission of J1741-302, and its corresponding in the Fermi-LAT data, is originated by the CR sea illuminating the cloud 8 at $260 \mathrm{pc}$ away from the GC.

\section{Conclusions}

We studied the cosmic ray population in the Galactic Center combining H.E.S.S. and FermiLAT data of the $\gamma$-ray emission from gas molecular clouds filling the region. In particular we scrutinized against those data the predictions of two different scenarios. The first - motivated by Fermi-LAT results - assuming that in the GC the Galactic CR sea is harder than in the rest of the Milky Way (gamma model), and the second in which a PeVatron is active at the GC position.

We showed that in the CMZ, due to the large uncertainties on the gas density distribution and not yet fully comprehended dynamical description of the central region, in addition with the limited extension of that area, we cannot discriminate among those models both giving a good description of the data. Therefore we addressed our attention to a massive cloud laying more than $200 \mathrm{pc}$ away from the GC, which was proposed to be the origin of the J1741-302 emission discovered by H.E.S.S.

Using PASS8 Fermi-LAT data we found, for the first time, that the hard spectrum found by H.E.S.S. for that source extends smoothly down to few GeVs. Then we shown that, while the gamma model reproduces both the slope and the normalization of that spectrum, the PeVatron scenario under-predicts the observed flux normalization by a factor of two.

This finding as well as the absence of a prominent features in the measured $\gamma$-ray spectrum over more than three energy decades - which should be present if the emission is due to a local component of $\mathrm{CR}$ acceleration in the GC, and takes over the background emission dominating at low energy - lead us to argue in favour of the hard CR sea interpretation of those measurements.

Further validations of that scenario should come from the planned CTA observation campaign of the GC region combined with the adoption of improved models of the 3-dimensional gas distribution.

\section{Acknowledgements}

We warmly thank Daniele Gaggero for the insightful discussions and providing the models and DRAGON outputs fundamental in the interpretation of our results. 


\section{References}

[1] F. Aharonian et al. [H.E.S.S. Collaboration], Nature 439 (2006) 695 doi:10.1038/nature04467 [astro-ph/0603021].

[2] A. Abramowski et al. [H.E.S.S. Collaboration], Nature 531 (2016) 476 doi:10.1038/nature17147 [arXiv:1603.07730 [astro-ph.HE]].

[3] H. Abdalla et al. [HESS Collaboration], arXiv:1706.04535 [astro-ph.HE].

[4] M. Aguilar et al. [AMS Collaboration], Phys. Rev. Lett. 114 (2015) 171103. doi:10.1103/PhysRevLett.114.171103

[5] https://fermi.gsfc.nasa.gov/ssc/data/access/lat/fl8y/FL8Y_ description_v5.pdf

[6] D. Gaggero, D. Grasso, A. Marinelli, M. Taoso and A. Urbano, Phys. Rev. Lett. 119, no. 3, 031101 (2017) doi:10.1103/PhysRevLett.119.031101 [arXiv:1702.01124 [astro-ph.HE]].

[7] C. Evoli, D. Gaggero, A. Vittino, G. Di Bernardo, M. Di Mauro, A. Ligorini, P. Ullio, D. Grasso, JCAP 02, 015, 2017 [arXiv:1607.07886 [astro-ph.HE]].

[8] D. Gaggero, A. Urbano, M. Valli and P. Ullio, Phys. Rev. D 91 (2015) no.8, 083012 doi:10.1103/PhysRevD.91.083012 [arXiv:1411.7623 [astro-ph.HE]].

[9] F. Acero et al. [Fermi-LAT Collaboration], Astrophys. J. Suppl. 223 (2016) no.2, 26 doi:10.3847/0067-0049/223/2/26 [arXiv:1602.07246 [astro-ph.HE]].

[10] M. Pothast, D. Gaggero, E. Storm and C. Weniger, JCAP 1810 (2018) no.10, 045 doi:10.1088/1475-7516/2018/10/045 [arXiv:1807.04554 [astro-ph.HE]].

[11] K. Ferriere, W. Gillard and P. Jean, A\&A 467 (2007) 611 doi:10.1051/0004-6361:20066992 [astro-ph/0702532].

[12] R. Z. Yang, D. I. Jones and F. Aharonian, A\&A 580 (2015) A90 doi:10.1051/0004-6361/201425233 [arXiv:1410.7639 [astro-ph.HE]].

[13] H. Abdalla et al. [H.E.S.S. and NANTEN Collaborations], A\&A 612 (2018) A13 doi:10.1051/0004-6361/201730581 [arXiv:1711.01350 [astro-ph.HE]].

[14] https://fermi.gsfc.nasa.gov/ssc/data/analysis/software/v11r5p3.html

[15] J. Kauffmann et al., A\&A 603 A89 (2017) A89 DOI: 10.1051/0004-6361/201628088. []arXiv: 1610.03499]. 